\title{
Well Differentiated Papillary Mesothelioma
}

National Cancer Institute

\section{Source}

National Cancer Institute. Well Differentiated Papillary Mesothelioma. NCI Thesaurus. Code C7635.

\begin{abstract}
A localized or multifocal mesothelioma arising from the peritoneum and less often the pleura. Cases arising from the peritoneum predominantly occur in women. It is characterized by the formation of papillae, covered by a single layer of blunt mesothelial cells. Mitotic figures are not present. There is no evidence of severe cytologic atypia. It has a relatively favorable clinical outcome, compared to malignant mesothelioma.
\end{abstract}

